\title{
Taxonomy of death
}

\section{Stephen Jay Gould}

Extinctions. Edited by Matthew H. Nitecki. University of Chicago Press: 1984. Pp.354. Hbk \$30, £27.50; pbk \$16, £14.75.

IN 1664, the great Jesuit scholar Athanasius Kircher published his Mundus Subterraneus, a massive treatise covering all objects found underground. The book is an aesthetic joy, but an intellectual puzzle to scientists of our time. It seems such a meaningless hodge-podge, a potpourri of unrelated objects - from dragons in caves, to fossils, to underground springs, to volcanoes - thrown together for the irrelevant reason that all lie beneath the Earth's surface. But Kircher's choice reflected neither stupidity nor expediency. Rather, his basic view of nature differed radically from ours, and taxonomies are the primary documents of changes in systems of explanation (I shall argue in a moment that the book here under review fails because its chosen taxonomy for death contravenes the probable causal structure of extinction as a biological phenomenon).

Kircher represents the last gasp of the pre-Newtonian non-mechanistic view of nature. He classified by common place and by similarity of form - conjunctions that had meaning in a world supposedly created at once to reflect God's transcendent good sense. A generation later, scientists had to classify by common genesis and common causal process - a criterion that made nonsense of an ordering by simple location underground.

In short, taxonomies are not neutral hatracks for the pristine facts of nature. They are theories that create and reflect the deep structure of science and human culture. A taxonomy is not just a ploy for convenient arrangement, but a hypothetical statement about the nature of things. This book, so admirable in nearly all parts taken separately, fails badly as an entity because its chosen taxonomy, seemingly obvious and "right" at first glance, is as superficial as Kircher's and for the same reason - it is based on a common appearance, not on common causes.

Extinctions is the latest of several volumes edited by Matthew $\mathrm{H}$. Nitecki and based on the annual Spring Systematics Symposia at the Field Museum in Chicago. The book, loosely ordered by descending scale, includes articles on mass extinction (A. Knoll on its non-occurrence in plants, S. Stanley on temperature as a dominant cause), species extinction in geological time (A. Walker on the death of robust australopithecines, P. Martin on Pleistocene mammals), and extinction, local and permanent, in ecological time (J. Diamond on isolated populations, B. Patterson on mammals in mountain refugia and
T. Lovejoy et al. on Amazonian forests).

Death seems so obvious as a unifying theme, given its inevitability, and its great ineluctable common principle - you ain't there any more after it's over. But is death a unitary phenomenon? Is it a proper basis for a coherent taxonomy? In an honest prefatory essay, D.M. Raup writes:

In summary, the several papers in this volume present an almost frightening array of conceptual frameworks and interpretations of extinction. It is clear that there is little agreement on the basics.... Whereas there is always controversy in science, I submit that the range of views presented in this volume shows a level of disparity or downright confusion which is of fscale.

I suggest that the reason for such maddening diversity is true disparity, not confusion. Maybe extinction is like being underground - an improper joining of disparate objects grouped by a superficial similarity judged either irrelevant or even misleading by our best modern notion of causes. Maybe death in mass extinction is so different from death in the everyday struggle for existence that the simple fact of disappearance records no meaningful basis for common classification. But why have we so automatically considered extinction as a coherent "thing" across all scales of time and magnitude?

In its claim to encompass all scales, conventional evolutionary theory (the Darwinian modern synthesis) rests on a principle of reduction and extrapolation natural selection is a struggle among organisms for reproductive success, and all phenomena of greater scale represent this process smoothly extended into larger and larger stretches of time. (Darwin's major statement about the geology of extinction, Chapter 10 in the Origin of Species, is one long argument for the continuationist vision - a claim that numbers and geographical range gradually dwindle before extinction and that the death of species is, therefore, a simple accumulation of individual losses in ordinary selection.)

\section{IMAGE \\ UNAVAILABLE \\ FOR COPYRIGHT REASONS}

Eighteenth-centur impact theory - the frontispiece to Vol. I of Buffon's Histoire Naturelle, showing God hurling a comet at the Sun to form the planets. 